\title{
SÉDIMENTATION DU SPERME ET SEX-RATIO CHEZ LES BOVINS
}

\author{
M. COUROT et C. ESNAULT \\ avec la collaboration technique de J.-C. TOURnEur et J.-C. Nicolde (1) \\ Laboratoire de Physiologie de la Reproduction, \\ Centre de Recherches de Tours, I. N. R. A. \\ Nouzilly BP 1, 37380 Monnaie
}

\section{RÉSUMÉ:}

Le fractionnement des éjaculats de taureau par sédimentation des spermatozoïdes (méthode de SCHILling) ne permet pas d'obtenir une déviation systématique du sex-yatio des veaux nés d'insémination artificielle après utilisation des fractions lourdes ou légères de l'éjaculat. D'autres facteurs que la seule différence de taille des spermatozoïdes due aux chromosomes X ou Y doivent intervenir au cours de la sédimentation.

\section{INTRODUCTION}

Plusieurs méthodes ont été proposées pour contrôler le sex-ratio des mammifères, soit par traitement endocrinien ou nutritionnel des femelles (Sulov, r966; STOL,KOWSKI et EMMERICH, I97I), soit par traitement in vitro de la semence des mâles pour tenter de séparer les spermatozoïdes porteurs respectivement du chromosome X ou Y (cf. revue de BEATTY, I970).

Dans ce dernier cas, outre des additifs divers dans le sperme (VLADIMIRskaja et Petreinko, I970; Schilling et PeTAC, I972), deux types de techniques ont surtout été utilisées, l'application d'un champ électrique (travaux inspirés de ceux de SCHRODER, I94I), et la sédimentation des spermatozoïdes dans un milieu approprié.

(1) Travail réalisé avec l'aide de la Coopérative d'Élevage du Sud-Ouest ( 8 I - Soual) et la collabora tion de l'U. N. C. E. I. A., M. Tourneur. 
Celle-ci pouvait faire appel à une centrifugation (LINDHAL, I960) ou bien se fonder sur la seule gravité (BHATTACHARYA, I962). C'est avec cette dernière technique que les résultats les plus nets ont été obtenus chez les bovins (ScHmLING, I965 et suite ; KNAACK, I968; KRZANOWSKI, I970). Les éjaculats de taureau peuvent ainsi être subdivisés en fractions " légère " et " lourde " suivant la manière dont les spermatozoïdes ont sédimenté dans le dilueur donné. Cette propriété va de pair avec certaines caractéristiques des spermatozoïdes : il a été observé que ceux de la fraction légère étaient d'une taille un peu inférieure (SchILING et al., I967 ; KNAACK, I968 ; EsNAULT et al., I970) et semblaient contenir un peu moins d'ADN, de protéines nucléaires (ESNAULT et al., I970) et d'arginine (SchilıING et Uzunov, I970) que les spermatozoïdes de la fraction lourde. C'est la fraction lourde qui donne une déviation du sex-ratio en faveur des femelles (KNAACK, Ig68 ; KRZANOwSKI, I970) et la fraction légère en faveur des mâles (KNAACK, I968). Or, le chromosome $Y$, déterminant le sexe mâle, est d'une taille très inférieure à celle du chromosome X (BAKER et al., I965).

Les différents arguments sur la dimension des chromosomes sexuels, la teneur en $\mathrm{ADN}$ et la déviation du sex-ratio dans les expériences sur bovins apparaissaient donc cohérents. Toutefois, en raison de certains échecs enregistrés avec cette méthode chez le lapin (BEDFORD et BIBEAU, I967; SchILLING, I97I) et compte tenu du fait que parmi les résultats rapportés sur bovins, une seule expérience (KNAACK, I968) a donné un excédent statistiquement significatif de veaux mâles avec la fraction "légère ", on pouvait se demander si le chromosome sexuel était réellement le facteur déterminant la manière dont sédimentaient les spermatozoïdes. Pour le vérifier, nous avons réalisé deux expériences avec le sperme de taureau, dans un centre d'insémination artificielle. Nous nous proposions de vérifier la répétabilité de la méthode en nous fondant sur le sex-ratio des veaux nés après utilisation de la semence traitée.

\section{MATÉRIEL, ET MÉTHODES}

Le sperme de 8 taureaux a été utilisé dans la première expérience, 2 Charolais, I Brun, 3 Blonds d'Aquitaine, 2 Limousins, et de 15 taureaux dans la seconde expérience, 2 FFPN, I Brun, 2 Blonds d'A quitaine et Io Limousins. La récolte et la vérification de la qualité étaient pratiquées suivant la technique en usage au Centre : motilité globale au moins égale à 4, concentration en spermatozoïdes supérieure à $\mathbf{I} \cdot 10^{\%} / \mathrm{ml}$. Les éjaculats - I ou 2 par séance de récolte hebdomadaire et $\mathbf{I}$ à 4 séries de récoltes par taureau selon les cas - étaient aussitôt dilués à concentration constante, $0,5 \cdot 10^{9} \mathrm{Spz} / \mathrm{ml}$ dans le milieu de Schiling (197I) refroidis et mis à sédimenter au froid dans un dilueur de densité appropriée, adapté du précédent après essai pour chaque éjaculat. Les différentes fractions étaient récupérées par soutirage après $60 \mathrm{mn}$ de sédimentation. Le nombre de spermatozoïdes présents dans chaque fraction était déterminé par comptage sur la cellule de Thoma. Les fractions homologues en fonction du profil de sédimentation (fig. I) étaient alors rassemblées et traitées pour être congelées en paillettes $\left(50 \cdot 10^{6}\right.$ spermatozoïdes totaux par dose $)$ : glycérolisation, équilibration et congélation rapide dans les vapeurs d'azote. L'ensemble des différentes fractions a présenté un taux de reviviscence après dégel, saffisant pour être utilisable en insémination artificielle. En outre, des éjaculats " témoins" ont été préparés selon la technique courante du Centre dans les quinze jours qui ont précédé et suivi la seconde période " expérimentale ". Les doses de semence ont été utilisées aussi bien pour des IA premières que pour des IA de retours. teurs.

Le sexe des veaux nés de ces traitements a été indiqué par les éleveurs ou par des insémina- 


\section{RÉSULTATS}

La courbe de répartition des spermatozoïdes dans la colonne de sédimentation a le plus souvent été bimodale (fig. I). Contrairement à SchILLING ou KNAACK, nous avons obtenu des spermatozoïdes dans les premières fractions soutirées, donc les plus lourdes (A). Ensuite, un pic à peu près symétrique était décelé dans lequel nous avons isolé la fraction lourde $(B)$, la fraction moyenne $(C)$ et la fraction légère $(D)$. Dans la première expérience, $\mathrm{B}$ et $\mathrm{C}$ ont été récoltées ensemble.

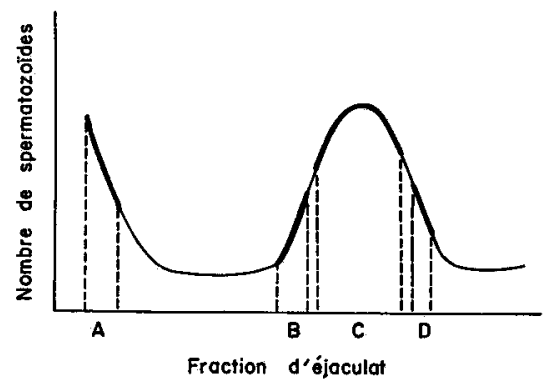

FIG. I

Dans l'expérience 1, 543 inséminations ont été réalisées (tabl. I a). La fécondance du sperme ainsi traité s'est révélée peu différente de la normale pour la fraction moyenne (69 p. Ioo NR à 60-9o j, 243 IA) et la fraction légère (64 p. Ioo, 86 IA), significativement moins bonne pour la fraction la plus lourde (53 p. Ioo, II4 IA, $\mathrm{P}<0,02)$. Ceci est sans relation avec le taux de reviviscence des spermatozoïdes après dégel. Cependant, il faut souligner l'écart important entre les taux de non-retour et les taux de vêlage correspondants, en raison des ennuis de reproduction au-delà de 60-9o $\mathrm{j}$ après IA (Io p. Ioo : retours en chaleur tardifs, avortements). et de la disparition d'animaux avant le vêlage ( 7 p. roo : accidents, ventes...)

L'effet du traitement sur le sex-ratio n'est pas net; dans tous les lots il y a un excédent de mâles. Avec les spermatozoïdes de la fraction légère ayant peu sédimenté (D), il y a une tendance à l'augmentation des mâles parmi les veaux nés : I 46 mâles/ Ioo femelles, ce qui équivaut à 59,3 mâles et 40,7 femelles pour Ioo veaux, mais le nombre de vêlages (86) est trop faible pour que l'écart observé soit statistiquement significatif. Les spermatozoïdes de la fraction moyenne (C) donnent un sex-ratio équivalent à celui que l'on trouve normalement sur un grand nombre d'animaux : Io6 mâles/Ioo femelles (SALISBURY et VAN DEMARK, I96I). Les spermatozoïdes qui ont sédimenté le plus vite (A) ne donnent pas d'excédent de veaux femelles.

Dans l'expérience 2, 862 IA témoins et 2230 IA expérimentales ont été réalisées. La fécondance n'a pu être mesurée que de façon globale : elle s'est révélée inférieure à la moyenne générale du Centre pour la période considérée, et un peu moins bonne pour la semence traitée que pour la semence témoin. Nous avons à nouveau observé un écart important entre le taux de non-retour et les taux de vêlage correspondants. 
L'analyse du sex-ratio (tabl. I b) montre qu'il n'y a aucune différence statistiquement significative par rapport à la moyenne générale théorique (106 mâles/ Ioo femelles, cf. supra). A l'exception de la fraction $\mathrm{C}$, les diverses séries témoins et expérimentales montrent une tendance à la prédominance de mâles. Mais cette fois, c'est dans la fraction lourde de l'éjaculat $(B)$ que se manifeste le plus fort excès de mâles (I35 mâles/Ioo femelles) qui n'est cependant pas statistiquement significatif.

\section{TABLEAU I}

Effet de la sédimentation des éjaculats de taureau sur leur pouvoir fécondant et sur le sex-ratio des veaux au moment de la naissance

\begin{tabular}{|c|c|c|c|c|c|}
\hline \multicolumn{6}{|c|}{ a) Expérience 1} \\
\hline Fractions ( $\left.{ }^{1}\right)$ & Témoins & A & $\mathrm{B}$ & $\mathrm{C}$ & $\mathrm{D}$ \\
\hline Nbre inséminations .... & - & 114 & - & 243 & 186 \\
\hline$\%$ NR $60-90$ j. $\left({ }^{2}\right) \ldots \ldots$ & - & $53,3^{*}$ & - & 68,7 & 64,0 \\
\hline Nbre veaux contrôlés. . & - & 48 & - & 118 & 86 \\
\hline Mâles $/ 100$ femelles $\left({ }^{3}\right)$. . & - & 118 & - & 107 & 146 \\
\hline
\end{tabular}

b) Expérience 2

\begin{tabular}{|c|c|c|c|c|c|}
\hline Nbre inséminations .... & 862 & 344 & 440 & 1124 & 322 \\
\hline Nbre veaux contrôlés .. & 481 & 134 & 176 & 488 & 124 \\
\hline Mâles $/ 100$ femelles $\left({ }^{3}\right)$ & 122,5 & 113 & 135 & 100 & 110 \\
\hline
\end{tabular}

(1) Fractions d'éjaculats séparées selon la vitesse de sédimentation des spermatozoides (cf. fig. 1) ; A : fraction la plus rapide, D : fraction la plus lente

(2) Le taux de non-retour à $60-90 \mathrm{j}$. pour l'ensemble du centre était de 71 p. 100 pour le mois pendant lequel ont été réalisées les IA expérimentales.

(3) Aucune différence significative avec la moyenne quel que soit le lot considéré.

* Significativement différent du p. $100 \mathrm{NR}$ de la fraction $\mathrm{C}(\mathrm{P}<0,02)$.

\section{DISCUSSION ET CONCLUSION}

Ainsi, au cours de deux expériences successives où la séparation des spermatozoïdes porteurs du chromosome $\mathrm{X}$ ou du chromosome $\mathrm{Y}$ a été tentée selon le principe d'une sédimentation différentielle (BATTACHARYa, I962), on n'a pas obtenu de déviation significative et systématique du sex-ratio. L a tendance à obtenir un excès de mâles s'est manifestée de façon aléatoire dans la fraction légère $(D)$ ou la fraction lourde (B) des éjaculats. On doit donc admettre qu'en dépit d'une hypothèse de départ qui semblait cohérente et que diverses publications tendaient à vérifier (ScHILING, I965 à I97I ; KNAACK, I970 ; KRZANOwSKI, I968), la séparation des spermatozoïdes 
de taureau ne résultait pas, dans nos expériences, de la seule différence de taille ou de poids due aux chromosomes sexuels. Ce résultat, déjà suggéré par KRALLINGER (I928), BEATTY (I970), BAHR (I970), VAN DUIJN et VAN VoORST (I97I), se trouve confirmé dans l'analyse théorique publiée récemment par RoBER'Ts (1972) : pour cet auteur, il n'est pas possible de séparer les spermatozoïdes porteurs des chromosomes $\mathrm{X}$ et $\mathrm{Y}$ sur la seule différence de taille des spermatozoïdes résultant de ces chromosomes car la variation relative de vitesse de sédimentation due à cette différence (I p. IOo) est très inférieure à celle observée dans les expériences pratiques (20 p. Ioo dans 1'expérience de KRzANOwsKI, I970). Cette faculté de sédimentation différentielle serait plus vraisemblablement reliée à la variabilité de taille des spermatozoïdes; or celle-ci est significative (Schilling et al., I967; KNAACK, I968; EsNaUlit et al., I970; VAN DUIJN et VAN VooRsT, I97I). Elle pourrait aussi dépendre du poids spécifique des spermatozoïdes qui varie avec les conditions de maturation et de stockage épididymaires, et donc avec la fréquence de collecte du sperme (LAvon et al., I97I). Toutefois, après fractionnement de l'éjaculat, et malgré l'obtention de courbes de séparation identiques à celle de la figure $\mathrm{I}$, les mesures microspectrophotométriques n'ont pas toujours permis de montrer des différences dans la teneur en ADN entre spermatozoides des fractions lourdes et légères (ESNAULT, I97I, non publié), contrairement à ce que nous avions obtenu dans une expérience antérieure (ESNAULt et al., I970). Par ailleurs, il n'a pas été possible de séparer deux populations différentes de spermatozoïdes par centrifugation en gradient de densité (O'DonneLl et Symons, I970). De toutes manières, d'autres données inconnues subsistent dans ce domaine car les vitesses de sédimentation effectives sont très supérieures à celles que permettent de calculer les lois qui régissent ce phénomène.

Ceci explique vraisemblablement les nombreuses causes d'échec rencontrées dans les tentatives de séparation par gravité des spermatozoïdes devant engendrer des mâles ou des femelles. D'autres méthodes doivent être mises en œutvre pour résoudre ce problème.

Ręu pour publication en mars 1973.

\title{
REMERCIEMENTS
}

Les auteurs tiennent à remercier le personnel de la Coopérative d'Élevage du Sud-Ouest pour la part prise dans l'exécution pratique de ce travail, fourniture de la semence, inséminations et recueil des résultats. Ils remercient en outre Mme Daveau et Mue Pisselet (I. N. R. A.) de l'aide apportée à diverses étapes de la préparation et la réalisation de ces expériences.

\section{SUMMARY}

\author{
SEDIMENTATION OF SPERMATOZOA AND SEX RATIO IN THE BOVINE
}

A systematic modification of the sex ratio was not obtained in calves born to AI with spermatozoa from the heavy and light fractions of ejaculates allowed to sediment (Schilling's method). Sedimentation must be influenced by other factors than simply the difference in size of spermatozoa bearing $\mathrm{X}$ or $\mathrm{Y}$ chromosomes. 


\section{RÉFÉRENCES BIBLIOGRAPHIQUES}

BAHR G.-F., r97r. Separation of $\mathrm{X}$ and $\mathrm{Y}$ bearing spermatozoa by gravity : a reconsideration. Sex." Ratio at birth. Prospects for Control. 1970 symposium. Am. Soc. Anim. Sci., 28-37.

Baker F.-N., Salisbury G.-W., Fechheimer N.-S., 1965. Measurements of bovine Chromosomes. Nature, 208, 97.

Beatty R.-A., I97o. The genetics of the mammalian gamete. Biol. Rev., 45, 73-II9.

Bhattacharya B.-C., Ig62. Die verschiedene Sedimentationsgeschwindigkeit der X- und Y-Spermien und die Frage der willkürlichen Geschlechtsbestimmung. Z, Wiss. Zool., 166, 203-250.

Esnault C., Ortavant R., Schilling E., r97o. Vatiations dans la teneur en ADN de deux populations de spermatozoïdes de Taureau séparées par sédimentation. Ann. Biol. anim. Bioch. Biophys., 10, 309-312.

KNAACK J., I968. I. Beitrage zum Spermiendimorphismus beim Rind. II. Willkürliche Geschlechbeeinflussung durch sedimentierte Rinderspermien. Fortpfl. Haust., 4, 256-269 et 279-282.

KRALLINGER E., 1928. Gibt es einem Spermatozoendimorphismus beim Haus rind? Thèse (cité par KNAAC, I968).

KRZANOwSKI M., I 97o. Dependance of primary and secondary sex-ratio on the rapidity of sedimentation of bull semen. $J$. Reprod. Fert., 23, I I-20.

Lavon U., Volcani R., Danon D., I971. An attempt to separate ejaculated bull spermatozoa into group in order to improve fertility. Sex-ratio at birth. Prospects for Control. 1970 symposium. Am. Soc. Anim. Sci., 19-27.

Lindhal P.-E., I960. Experimental influence upon the distribution of the sexes in mammals by separation of male and female determining spermatozoa. Z. Tierz. Züchtungsbiol., 74, I8 I-I97.

O'Donnell J.-M., Symons D. B. A., I970. Centrifugal patterns and morphological variation of bull spermatozoa. J. Reprod. Fert., 23, 4ז-48.

Roberts A.-M., I972. Gravitational separation of X and $\mathrm{Y}$ spermatozoa. Nature, 238, $223-225$.

Salisbury G.-W., VAN Demark N.-L., I96I. Physiology of reproduction and artificial insemination of cattle. Freeman et Co. Ed.

Schilling E., 1965. Experimentelle Untersuchungen zur Geschlechtsbeeinflussung beim Rind. Natureviss., 12,353 .

Schilling E., JazBec I., Schmid P., I967. Grösse und Geswindigkeit der Samenzellen vom Rind und Schaf und deren mögliche Beziehungen zum Geschlecht. Züchtungsbiol., 83, 33I-339.

Schilling E., Uzunov G., I97o. Stickstoffgehalt und Aminosaüren ir schweren und leichten Samenzellen vom Rind und Schaf. Zuchthygiene, 3, 104-112.

Schilling E., I97x. Sedimentation as an approach to the problem of separating X-and Y-chromosome-bearing spermatozoa. Sex-Ratio at birth. Prospects for Control. 1970 symposium. Am. Soc. Anim. Sci., 76-84.

Schilling E., Petac D., I972. Treatment of semen with chemicals and sex-ratio in rabbits and swine. Abstr. VII ${ }^{\mathrm{e}}$ Cong. intern. Reprod. anim., Munich, p 46.

Schroder V., I94I. Künstliche geschlechtsregulation der Nachkommenschaft der Saügetiere und ihre biologische Kontrolle. Z. Tierzucht. Zuchtungsbiol., 50, x-I5.

Stolkowski J., Eмmerich E., I97I. Influence de la nutrition minérale de la vache sur la répartition des sexes dans la descendance : une enquête prospective, un essai expérimental. Ann. Endocr., 32, $3-15$.

Sulov V.-V., Ig66. Regulating the formation of sex in the progeny of sheep (en russe). Sel'. Khoz. Nauki. Alma. Ata., 11, 44-48.

Van Duijn Jr. C., Van Vookst C., r97r. Precision measurements of dimensions, refractive index and mass of bull spermatozoa in the living stage. Mikroskopie, 27, 142-167.

Vladimirskaja E.-M., Petrenko I.-P., I97o. On the phenotypic demarcation in mass and size of gametes in relation to the problem of sex regulation in cattle (en russe). Genetika S. S. S. R., 6, 73-87. 